\title{
The impact of initial statin treatment decisions on cardiovascular outcomes in clinical care settings: estimates using the Archimedes Model
}

This article was published in the following Dove Press journal:

ClinicoEconomics and Outcomes Research

9 November 2012

Number of times this article has been viewed

\author{
Andrew van Herick' \\ C Andy Schuetz' \\ Peter Alperin' \\ Michael F Bullano² \\ Sanjeev Balu ${ }^{2}$ \\ Sanjay Gandhi ${ }^{2}$
}

'Archimedes, Inc, San Francisco, CA, USA; ${ }^{2}$ AstraZeneca Pharmaceuticals LP, Wilmington, DE, USA
Correspondence: Andrew van Herick I 23 Mission St, I Ith Floor, San Francisco, CA 94105, USA

$\mathrm{Tel}+\mathrm{I} 4154900416$

Fax + I 4154900399

Email andrew.vanherick@

archimedesmodel.com
Purpose: Many patients treated for dyslipidemia do not achieve recommended cholesterol goals despite the widespread availability of effective statins. Pharmaceutical claims show a strong tendency for patients to remain on their initially assigned treatment. With computer simulations, the impact of initial statin treatment decisions on medium- and long-term cardiovascular outcomes were examined.

Patients and methods: Using the Archimedes Model, three treatment scenarios were simulated. Patients initiated treatment with simvastatin $(20,40$, or $80 \mathrm{mg})$, atorvastatin $(10,20$, 40 , or $80 \mathrm{mg}$ ), or rosuvastatin $(10,20$, or $40 \mathrm{mg})$, and periodically intensified treatment. The simulated population consisted of 50,025 patients, aged 45-70 years, with low-density lipoprotein cholesterol exceeding goal. The proportion of patients initiating each dose was calibrated to United States pharmacy claims. Patients not reaching goal intensified the dose of their current statin or switched to an appropriate dose of rosuvastatin at rates matching pharmacy claims. Biomarkers and major adverse cardiovascular events (MACE) were tracked for 10 years and several high-risk subpopulations were analyzed. Statin models used biomarker effects from the STELLAR (Statin Therapies for Elevated Lipid Levels Compared Across Doses to Rosuvastatin) trial and outcomes data from various trials.

Results: Initiating therapy with rosuvastatin reduced MACE more than simvastatin or atorvastatin. The 5- year relative risk of MACE was 0.906 (95\% confidence interval: 0.888-0.923; $P<0.001)$ for initial treatment with atorvastatin rather than simvastatin, $0.831(0.812-0.850$; $P<0.001)$ for rosuvastatin rather than simvastatin, and $0.918(0.898-0.938 ; P<0.001)$ for rosuvastatin rather than atorvastatin. Subgroups with higher MACE incidence experienced greater absolute benefit.

Conclusion: Considering observed rates of treatment intensification, initial treatment choices appear to significantly impact medium- and long-term cardiovascular risk. Patients at high cardiovascular risk are good candidates for aggressive initial therapy.

Keywords: rosuvastatin, atorvastatin, simvastatin, simulation, modeling

\section{Introduction}

Each year, cardiovascular disease (CVD) causes an estimated 17 million deaths globally. ${ }^{1}$ Elevated serum cholesterol is a modifiable risk factor that contributes significantly to cardiovascular risk. ${ }^{2}$ Statins represent a powerful therapeutic option for lowering cholesterol and preventing major adverse cardiovascular events (MACE). ${ }^{3}$ Despite the widespread availability of statins, a significant proportion of treated patients fail to reach their recommended low-density lipoprotein cholesterol (LDL-C) goals. ${ }^{4,5}$ Evidence from studies analyzing pharmaceutical claims data suggests that conservative initial treatment combined with low subsequent titration rates may partially account for 
this observation. ${ }^{4,5}$ In particular, these studies show a strong tendency among patients to remain on their initial therapy despite inadequate goal attainment, suggesting an opportunity to reduce medium- and long-term cardiovascular risk by initiating statin therapy using more efficacious statins.

To better understand the cardiovascular impact of initiating statin therapy at clinically relevant doses, this study used the Archimedes Model to simulate a series of clinical trials comparing the relative effectiveness of three different statin titration protocols, reflective of real-world care, in which patients received conservatively assigned, label-indicated statin doses, and subsequently intensified treatment at rates determined from United States pharmacy claims data. In the first scheme, patients initiated therapy using label-indicated starting doses of simvastatin - a commonly prescribed generic statin, but not the most powerful generic option as defined by LDL-C reduction per milligram dose. In the second scheme, they initially received atorvastatin - a potent statin currently available in generic form. In the third scheme, they initially received rosuvastatin.

\section{Methods}

\section{The Archimedes Model}

The Archimedes Model is a trial-validated, clinically detailed simulation model of human physiology, disease progression, and health care delivery. ${ }^{6,7}$ The core of the Model is a set of algebraic and differential equations representing the physiological pathways pertinent to diseases and their complications. The model currently includes coronary artery disease; diabetes and its complications; congestive heart failure; stroke, hypertension; colorectal, lung, and breast cancers; and more in a single integrated model. Use of a single model enables Archimedes to compare a wide range of treatments, guidelines, and performance measures within an integrated system, and to address comorbidities, syndromes that span multiple organ systems, drugs that have multiple effects, and combinations of treatments. The use of continuous equations preserves the continuous nature of biological variables and the interactions between them, as well as the continuous nature of chronic disease. Interventions, both to prevent diseases and to manage them when they occur, are modeled at the level of the underlying biology.

The Archimedes Model is well suited to simulating clinical trials, allowing interventions and protocol changes to be compared using an identical population for each trial arm. First, a study cohort of virtual patients meeting the trial inclusion/exclusion criteria is created by generating simulated individuals with individual characteristics observed in real-world patients (in the present case, patients observed in the National Health and Nutrition Examination Survey 1999-2006 were used). ${ }^{8}$ Using person-specific data from a real population, the Archimedes Model creates simulated populations of virtual individuals with distributions and correlations of risk factors, behaviors, medication usage, and medical histories reflective of the real population. Each patient's virtual life is then simulated for the trial period. Each person has a simulated physiology that can begin to function abnormally (due to the onset of a disease), triggering signs and symptoms (such as angina in the case of coronary heart disease). The virtual patients then may seek care in virtual outpatient clinics or emergency rooms. Virtual patients seeking care will receive virtual diagnoses and be treated with virtual treatments, all reflective of real-world health care protocols and guidelines.

The accuracy of the Archimedes Model is verified using a variety of techniques, most importantly the simulation of clinical trials, comparing the model's results with the observations from the trials. More than 50 major clinical trials have been used to validate the model, including several landmark statin trials (HPS [Heart Protection Study], ${ }^{9}$ 4S [Scandinavian Simvastatin Survival Study], ${ }^{10}$ IDEAL [Incremental Decrease in End Points Through Aggressive Lipid Lowering], ${ }^{11}$ CARDS [Collaborative Atorvastatin Diabetes Study], ${ }^{12}$ TNT [Treating to New Targets], ${ }^{13}$ and others) that are directly relevant to the current study. More details on the model can be found on the Archimedes website (http://www.archimedesmodel.com) and in prior Archimedes publications. ${ }^{7,14}$

\section{Simulated study population}

The aim of this simulation was to compare the effectiveness of statin titration protocols in a patient cohort eligible for statin therapy. A virtual population was created based on actual individuals randomly drawn from the National Health and Nutrition Examination Survey 1999-2006 database. ${ }^{8}$ Virtual candidates were considered eligible for enrollment if they were aged 45-70 years and had LDL-C exceeding their conservative individual Adult Treatment Panel (ATP)-III goal (LDL-C targets of 100, 130, and $160 \mathrm{mg} / \mathrm{dL}$ ) ${ }^{15}$ after a washout period in which all lipid-lowering therapies were withheld. The benefit of treatment was analyzed for the full cohort and for a series of embedded subpopulations: those with diabetes mellitus; those with prior CVD - defined as a history of myocardial infarction or stroke; those with LDL-C more than double their aggressive ATP-III goal; those classified as moderate risk under standard ATP-III 
guidelines; and those classified as high risk under standard ATP-III guidelines.

\section{Simulation design}

The simulation was structured as a three-armed clinical trial comparing initial treatment with simvastatin, atorvastatin, and rosuvastatin. In the simvastatin arm, patients received a 20-, 40-, or 80-mg dose of simvastatin; in the atorvastatin arm, a 10, 20, 40, or $80 \mathrm{mg}$ dose of atorvastatin; and in the rosuvastatin arm, a 10, 20, or $40 \mathrm{mg}$ dose of rosuvastatin. Exactly the same virtual patients were simulated in each arm. Treatment in all trial arms began with a washout period in which all lipid-lowering therapies prescribed prior to trial enrollment were discontinued. Following the washout, simulated patients were prescribed a dose of the statin corresponding to the arm. In order for the simulation to realistically reflect current patient care, initial treatments were assigned using an algorithm that sought to minimize overtreatment while matching the distributions of initial dose assignments observed in the pharmacy claims data. Patient compliance with the initial treatments was calibrated to produce ATP-III LDL-C goal attainment rates and attenuated treatment effects consistent with prior publications. ${ }^{4,5}$ Compliance with the initial prescription was assumed to be independent of the statin offered, so the same compliance level was used in all three treatment arms. Once a patient complied with a statin prescription, they remained compliant with all subsequent prescriptions for the remainder of the simulation.

In follow-up visits, patients with LDL-C level greater than their target goal were considered eligible for treatment intensification. To reflect different treatment pathways for patients on the assigned statin treatment, a random draw assigned each patient to either (1) continue their current treatment, (2) increase the dose of the currently prescribed statin if a more powerful dose was available, or (3) switch to a dose of rosuvastatin 10,20 , or $40 \mathrm{mg}$, representing a modest treatment intensification from the current prescription. Those patients switching to rosuvastatin continued to be eligible for intensification using rosuvastatin until the maximum dose of $40 \mathrm{mg}$ was reached. The possibility of treatment intensification using rosuvastatin in all arms ensured a realistic titration protocol, as most real-world health plans offer patients access to the strongest branded agents if less efficacious agents prove insufficient. Patients received follow-up visits 6 weeks after trial start, and periodically thereafter (with frequency ranging from 5 months to 1 year, consistent with the ATP-III guidelines) for the first 5 years of the trial. Patients were managed precisely to their LDL-C goal (that is all patients with LDL-C exceeding goal were eligible for intensification). After 5 years, no further intensification was offered, and patients maintained their statin treatments until the end of the simulation.

Blood pressure and glucose were managed according to The Seventh Report of the Joint National Committee on Prevention, Detection, Evaluation, and Treatment of High Blood Pressure and American Diabetes Association guidelines, respectively. ${ }^{16,17}$ Compliance with these guidelines was set to match levels of medication use observed in the National Health and Nutrition Examination Survey.

\section{Modeling statin treatments}

The statin treatment models were based on published clinical trials. The general approach used to model statins for this comparison has been described in detail elsewhere. ${ }^{18}$ Briefly, in the Archimedes Model, statins reduce cardiovascular outcomes by affecting biomarker values (primarily through total cholesterol/high-density lipoprotein cholesterol ratio) and are parameterized with additional disease-specific benefits referred to as "pleiotropic" effects. Pleiotropic effects capture disease-specific benefits of interventions beyond those directly resulting from biomarker changes and are calibrated to match real-world trial results. The creation of each statin model began by matching the effects on the lipid panel to effects observed in the STELLAR (Statin Therapies for Elevated Lipid Levels Compared Across Doses to Rosuvastatin) trial. ${ }^{19}$ Pleiotropic effects on CVD risk were then calibrated using the published hazard ratios and/or relative risks (RRs) from clinical outcomes trials. In cases in which a credible trial for a particular dose existed, the pleiotropic effect was calibrated directly based on the disease risk reductions observed in the trial. Otherwise, the expected RR for the statin dose was estimated based on a linear regression using other doses of the same statin. The observed total cholesterol/ high-density lipoprotein cholesterol reduction for each dose from the STELLAR (Statin Therapies for Elevated Lipid Levels Compared Across Doses to Rosuvastatin) trial served as the independent variable in the regression. Table 1 shows an overview of all statin models used in this study along with the clinical trials that supported each modeling effort. Figure 1 shows the relationship of the statin models to the results of published clinical trials.

\section{Efficacy outcomes}

The primary outcome was MACE, defined as the first occurrence of fatal or nonfatal myocardial infarction, fatal or nonfatal stroke, or death from cardiovascular causes. 
Table I Summary of statin models. Relative lipid panel changes are based on the STELLAR (Statin Therapies for Elevated Lipid Levels Compared Across Doses to Rosuvastatin) trial ${ }^{19}$

\begin{tabular}{|c|c|c|c|c|c|c|}
\hline \multirow[t]{2}{*}{ Intervention } & \multicolumn{3}{|c|}{ Relative lipid panel change* } & \multicolumn{2}{|l|}{$\mathbf{R R}$} & \multirow[t]{2}{*}{ Model data sources } \\
\hline & LDL-C & TC & HDL-C & MI & Stroke & \\
\hline \multicolumn{7}{|l|}{ Simvastatin } \\
\hline $20 \mathrm{mg}$ & $-35.0 \%$ & $-25.7 \%$ & $5.7 \%$ & 0.64 & 0.69 & $4 S^{10}$ \\
\hline $40 \mathrm{mg}$ & $-38.8 \%$ & $-27.9 \%$ & $6.0 \%$ & 0.62 & 0.67 & HPS $^{9}$ \\
\hline $80 \mathrm{mg}$ & $-45.8 \%$ & $-32.9 \%$ & $6.5 \%$ & 0.56 & 0.60 & Regression ${ }^{\dagger}, 4 \mathrm{~S},{ }^{10} \mathrm{HPS}^{9}$ \\
\hline \multicolumn{7}{|l|}{ Atorvastatin } \\
\hline $10 \mathrm{mg}$ & $-36.8 \%$ & $-28.0 \%$ & $4.6 \%$ & 0.59 & 0.63 & CARDS, ${ }^{12}$ ASCOT $^{24}$ \\
\hline $20 \mathrm{mg}$ & $-42.6 \%$ & $-31.8 \%$ & $4.8 \%$ & 0.54 & 0.61 & Regression ${ }^{\dagger}$, CARDS,,$^{12}$ ASCOT, ${ }^{24} \mathrm{TNT}^{13}$ \\
\hline $40 \mathrm{mg}$ & $-47.8 \%$ & $-35.8 \%$ & $4.4 \%$ & 0.49 & 0.58 & Regression ${ }^{\dagger}$, CARDS,,$^{12}$ ASCOT, ${ }^{24} \mathrm{TNT}^{13}$ \\
\hline $80 \mathrm{mg}$ & $-51.1 \%$ & $-39.0 \%$ & $3.6 \%$ & 0.46 & 0.57 & CARDS, ${ }^{12}$ ASCOT,,$^{24} \mathrm{TNT}^{13}$ \\
\hline \multicolumn{7}{|l|}{ Rosuvastatin } \\
\hline $10 \mathrm{mg}$ & $-45.8 \%$ & $-32.9 \%$ & $7.7 \%$ & 0.50 & 0.53 & Regression ${ }^{\dagger}$, JUPITER, ${ }^{23}$ CARDS, ${ }^{12}$ ASCOT, $,{ }^{24} \mathrm{TNT}^{\ddagger}, 13$ \\
\hline $20 \mathrm{mg}$ & $-52.4 \%$ & $-37.6 \%$ & $9.5 \%$ & 0.44 & 0.49 & JUPITER ${ }^{23}$ \\
\hline $40 \mathrm{mg}$ & $-55.0 \%$ & $-40.2 \%$ & $9.6 \%$ & 0.41 & 0.47 & Regression ${ }^{\dagger}$, JUPITER, ${ }^{23}$ CARDS, ${ }^{12}$ ASCOT,,${ }^{24}$ TNT $^{\ddagger}, 13$ \\
\hline
\end{tabular}

Notes: *The relationship between lipids expressed by the Friedewald equation is a fundamental assumption of the Archimedes Model. Triglycerides are omitted because they were used as an independent variable to calibrate the other lipid changes and do not directly influence risk in the Model; ${ }^{\dagger}$ regression based on listed trials; ${ }^{\ddagger}$ non-rosuvastatin trials used because regression needed multiple data points and no other outcomes trials existed for rosuvastatin.

Abbreviations: ASCOT, Anglo-Scandinavian Cardiac Outcomes Trial; 4S, Scandinavian Simvastatin Survival Study; CARDS, Collaborative Atorvastatin Diabetes Study; HDL-C, high-density lipoprotein cholesterol; HPS, Heart Protection Study; JUPITER, Justification for the Use of Statins in Primary Prevention: An Intervention Trial Evaluating Rosuvastatin; LDL-C, low-density lipoprotein cholesterol; MI, myocardial infarction; RR, relative risk; TC, total cholesterol; TNT, Treating to New Targets.

The cumulative incidence of MACE for each trial arm was determined from Kaplan-Meier survival estimates. The relative effectiveness of each treatment scenario was evaluated using RR, and the absolute benefit was evaluated using the number needed to treat (NNT). The RR estimates were computed as the number of events in the exposure scenario divided by the number of events in the control scenario. The NNT values were derived from Kaplan-Meier survival estimates using the method of Altman and Andersen. ${ }^{20}$ Standard error computations for comparative statistics took into account the high correlation between trial arms, which results from simulating identical populations for each arm. This leads to smaller confidence intervals and $P$-values than might be expected for a similar real-world randomized study. All confidence estimates reflect the statistical uncertainty resulting from the stochastic nature of the Archimedes Model.

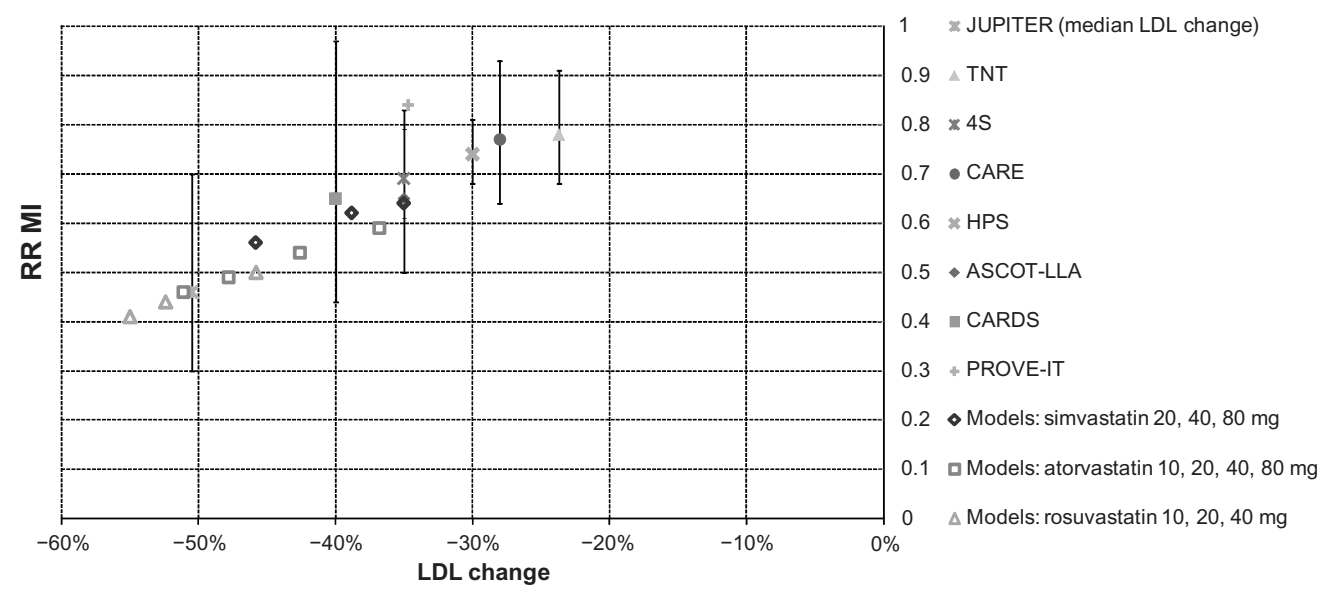

Figure I A comparison of the myocardial infarction risk benefit of the statin models with real-world clinical trial results plotted against relative change in low-density lipoprotein cholesterol.

Notes: $95 \%$ confidence interval not available for the PROVE-IT trial; ${ }^{22}$ trial comparisons: JUPITER, rosuvastatin 20 mg versus placebo; ${ }^{23}$ TNT, atorvastatin 10 mg versus $80 \mathrm{mg} ;{ }^{13} 4 \mathrm{~S}$, simvastatin $20 \mathrm{mg}$ versus placebo; ${ }^{10}$ CARE, pravastatin $40 \mathrm{mg}$ versus placebo; ${ }^{25} \mathrm{HPS}$, simvastatin 40 mg versus placebo; ${ }^{9}$ ASCOT-LLA, atorvastatin 10 mg versus placebo; ${ }^{24}$ CARDS, atorvastatin $10 \mathrm{mg}$ versus placebo; ${ }^{\prime 2}$ PROVE-IT, pravastatin $40 \mathrm{mg}$ versus atorvastatin $80 \mathrm{mg} .{ }^{22}$

Abbreviations: 4S, Scandinavian Simvastatin Survival Study; ASCOT-LLA, Anglo-Scandinavian Cardiac Outcomes Trial - Lipid Lowering Arm; CARDS, Collaborative Atorvastatin Diabetes Study; CARE, Cholesterol and Recurrent Events; HPS, Heart Protection Study; JUPITER, Justification for the Use of Statins in Primary Prevention: An Intervention Trial Evaluating Rosuvastatin; LDL, low-density lipoprotein; MI, myocardial infarction; PROVE-IT, Pravastatin or Atorvastatin Evaluation and Infection Therapy; RR, relative risk; TNT, Treating to New Targets. 
Scientific uncertainty associated with the modeling assumptions is explored through sensitivity analyses.

\section{Sensitivity analyses}

The interpretation of pharmaceutical claims data supporting this study involved several simplifying assumptions that potentially impact the conclusions drawn from this study. To examine the sensitivity of the findings to these assumptions, and to better understand the relationship between initial treatment decisions and the level of intensification administered through follow-up care, three additional clinical trials were simulated:

- Low-intensification case: patients not at goal were three times less likely to intensify treatment as in the main study.

- High-intensification case: patients not meeting goal were three times more likely to intensify treatment as in the main study.

- Atorvastatin-only case: patients initiating treatment with atorvastatin could only intensify treatment using atorvastatin.

Except for the rates of treatment intensification, the protocols and treatments used for the high- and low-titration cases were identical to those of the main study. In the atorvastatinonly case, patients intensified treatment at the same rates as in the main simulation, but without the possibility of taking rosuvastatin.

\section{Results \\ Population baseline characteristics}

The size of the virtual population was determined considering limits in computational resources and estimates of the sample size required to detect a difference in MACE incidence at 5 years with a power of 0.9. The baseline characteristics for the full cohort of 50,025 patients, along with those of the embedded subpopulations, are provided in Table 2.

\section{Statin treatment patterns}

To verify that the simulations reflected realistic clinical care with respect to both initial treatment assignments and follow-up care, statin use in the simulation was compared with trends observed in pharmaceutical claims data. At baseline, the proportion of patients receiving prescriptions for each initial statin dose closely matched the claims data (Table 3).

Table 2 Baseline characteristics of the overall population and embedded subpopulations

\begin{tabular}{|c|c|c|c|c|c|c|}
\hline Characteristic & All & DM & $\begin{array}{l}\text { ATP-III } \\
\text { moderate risk* }\end{array}$ & $\begin{array}{l}\text { ATP-III } \\
\text { high risk }^{\dagger}\end{array}$ & $\begin{array}{l}\text { LDL-C } 2 \times \\
\text { ATP-III goal }{ }^{\ddagger}\end{array}$ & Prior CVD \\
\hline$N$ & 50,025 & 12,793 & 16,262 & 23,430 & 15,830 & 4,738 \\
\hline Age (years) & $57(7)$ & $58(7)$ & $56(7)$ & $59(7)$ & $59(7)$ & $59(7)$ \\
\hline Men (\%) & 56 & 50 & 59 & 63 & 63 & 67 \\
\hline BMI $\left(\mathrm{kg} / \mathrm{m}^{2}\right)$ & $30(7)$ & $36(7)$ & $29(5)$ & $32(7)$ & $31(7)$ & $30(6)$ \\
\hline \multicolumn{7}{|l|}{ Blood pressure (mmHg) } \\
\hline Systolic & $129(17)$ & $128(16)$ & $130(16)$ & |31 (I8) & 131 (18) & 127 (19) \\
\hline Diastolic & $75(I I)$ & $73(\mathrm{II})$ & $77(12)$ & $74(12)$ & $75(12)$ & $72(12)$ \\
\hline \multicolumn{7}{|l|}{ Cholesterol (mg/dL) } \\
\hline $\mathrm{TC}$ & $252(50)$ & $236(47)$ & $245(37)$ & $245(57)$ & $280(6 I)$ & $24 I(53)$ \\
\hline LDL-C & $167(4 \mid)$ & $|5|(39)$ & $164(29)$ & $158(48)$ & $190(49)$ & 155 (44) \\
\hline HDL-C & $49(14)$ & $52(14)$ & $46(11)$ & $48(14)$ & $47(13)$ & $49(14)$ \\
\hline Triglycerides & $181(113)$ & $164(100)$ & $177(96)$ & $195(132)$ & $210(139)$ & $184(123)$ \\
\hline $\mathrm{HbA}_{\mathrm{Ic}}(\%)$ & $5.9(1.5)$ & $7.3(1.7)$ & $5.4(1)$ & $6.5(1.7)$ & $6.3(1.7)$ & $6(1.6)$ \\
\hline \multicolumn{7}{|l|}{ ATP-III risk category (\%) } \\
\hline High* & 47 & 100 & 0 & 100 & 88 & 99 \\
\hline Moderate ${ }^{\dagger}$ & 33 & 0 & 100 & 0 & 9 & I \\
\hline Low & 21 & 0 & 0 & 0 & 4 & I \\
\hline \multicolumn{7}{|l|}{ Medical history } \\
\hline Diagnosed diabetes (\%) & 26 & 100 & 0 & 55 & 43 & 24 \\
\hline Current smoker (\%) & 25 & 16 & 31 & 27 & 28 & 28 \\
\hline Hypertension (\%) & 58 & 72 & 62 & 67 & 68 & 54 \\
\hline $\mathrm{MI}(\%)$ & 6 & 6 & 0 & 12 & 11 & 59 \\
\hline Stroke (\%) & 4 & 3 & 0 & 9 & 7 & 44 \\
\hline Revascularization (\%) & 5 & 5 & 0 & 10 & 9 & 35 \\
\hline
\end{tabular}

Notes: Values represent mean (standard deviation) unless indicated as percentage; *includes those at moderate and moderately high cardiovascular risk as defined by guidelines; 'includes those at high and very high cardiovascular risk as defined by guidelines; "based on aggressive goals of 70 , 100 , and I 30 mg/dL defined in guidelines. Abbreviations: ATP-III, Adult Treatment Panel III; BMI, body mass index; CVD, cardiovascular risk; DM, diabetes mellitus; HbA ${ }_{1 \mathrm{c}}$, hemoglobin $\mathrm{A}_{\mathrm{Ic}}$; HDL-C, high-density lipoprotein cholesterol; LDL-C, low-density lipoprotein cholesterol; MI, myocardial infarction; TC, total cholesterol. 
Table 3 Comparison between patterns of initial dose assignment observed in pharmaceutical claims versus those in the simulation

\begin{tabular}{lll}
\hline Initial & \multicolumn{2}{c}{ Proportion prescribed treatment } \\
\cline { 2 - 3 } prescription & Simulation & Pharmacy claims \\
\hline Simvastatin & & \\
$20 \mathrm{mg}$ & $49 \%$ & $49 \%$ \\
$40 \mathrm{mg}$ & $43 \%$ & $42 \%$ \\
$80 \mathrm{mg}$ & $8 \%$ & $8 \%$ \\
Atorvastatin & & \\
$10 \mathrm{mg}$ & $36 \%$ & $36 \%$ \\
$20 \mathrm{mg}$ & $37 \%$ & $37 \%$ \\
$40 \mathrm{mg}$ & $21 \%$ & $21 \%$ \\
$80 \mathrm{mg}$ & $6 \%$ & $6 \%$ \\
Rosuvastatin & & \\
$10 \mathrm{mg}$ & $73 \%$ & $73 \%$ \\
$20 \mathrm{mg}$ & $23 \%$ & $23 \%$ \\
$40 \mathrm{mg}$ & $4 \%$ & $4 \%$ \\
\hline
\end{tabular}

At year one, the proportions of patients intensifying treatment likewise corresponded closely to the pharmaceutical claims. Both of these matches were expected, as these were direct calibration points of the simulation. However, the pattern of treatment intensification at year two was not directly calibrated, yet also closely matched the patterns observed in claims data, suggesting that simulated treatment patterns would likely remain consistent with real-world trends for subsequent time points. An overview of treatment intensification patterns for years one and two is shown in Table 4.

\section{Effects of treatments on lipids and goal attainment}

All three scenarios produced substantial improvements in the mean lipid levels. After 1 year, the mean reduction in total cholesterol/high-density lipoprotein cholesterol ratio from baseline was $25 \%$ for the simvastatin scenario, $28 \%$ for atorvastatin, and $31 \%$ for rosuvastatin. The corresponding reduction in LDL-C was 30\%, 34\%, and 38\%, respectively. An overview of biomarker effects is shown in Table 5. After 1 year, the proportion of patients meeting their conservative ATP-III LDL-C goal (LDL-C targets of 100, 130, and $160 \mathrm{mg} / \mathrm{dL}$ ) was $65 \%$ for those initially assigned simvastatin, $69 \%$ for atorvastatin, and $73 \%$ for rosuvastatin.

Table 4 Comparison of treatment intensification patterns observed in pharmaceutical claims versus those in the simulation

\begin{tabular}{|c|c|c|c|c|}
\hline \multirow[t]{2}{*}{ Initial treatment } & \multicolumn{2}{|c|}{ Increase dose } & \multicolumn{2}{|c|}{ Switch to rosuvastatin } \\
\hline & Simulation & Pharmacy claims & Simulation & Pharmacy claims \\
\hline \multicolumn{5}{|l|}{ Year I } \\
\hline \multicolumn{5}{|l|}{ Simvastatin } \\
\hline $20 \mathrm{mg}$ & $13 \%$ & $13 \%$ & $1 \%$ & $1 \%$ \\
\hline $40 \mathrm{mg}$ & $7 \%$ & $7 \%$ & $2 \%$ & $2 \%$ \\
\hline $80 \mathrm{mg}$ & $0 \%$ & $0 \%$ & $5 \%$ & $4 \%$ \\
\hline \multicolumn{5}{|l|}{ Atorvastatin } \\
\hline $10 \mathrm{mg}$ & $13 \%$ & $13 \%$ & $1 \%$ & $1 \%$ \\
\hline $20 \mathrm{mg}$ & $9 \%$ & $10 \%$ & $2 \%$ & $2 \%$ \\
\hline $40 \mathrm{mg}$ & $7 \%$ & $7 \%$ & $3 \%$ & $2 \%$ \\
\hline $80 \mathrm{mg}$ & $0 \%$ & $0 \%$ & $5 \%$ & $4 \%$ \\
\hline \multicolumn{5}{|l|}{ Rosuvastatin } \\
\hline $10 \mathrm{mg}$ & $11 \%$ & $11 \%$ & & \\
\hline $20 \mathrm{mg}$ & $9 \%$ & $9 \%$ & & \\
\hline $40 \mathrm{mg}$ & $0 \%$ & $0 \%$ & & \\
\hline \multicolumn{5}{|l|}{ Year 2} \\
\hline \multicolumn{5}{|l|}{ Simvastatin } \\
\hline $20 \mathrm{mg}$ & $18 \%$ & $18 \%$ & $2 \%$ & $2 \%$ \\
\hline $40 \mathrm{mg}$ & $10 \%$ & $10 \%$ & $3 \%$ & $3 \%$ \\
\hline $80 \mathrm{mg}$ & $0 \%$ & $0 \%$ & $7 \%$ & $6 \%$ \\
\hline \multicolumn{5}{|l|}{ Atorvastatin } \\
\hline $10 \mathrm{mg}$ & $17 \%$ & $18 \%$ & $1 \%$ & $2 \%$ \\
\hline $20 \mathrm{mg}$ & $13 \%$ & $14 \%$ & $3 \%$ & $3 \%$ \\
\hline $40 \mathrm{mg}$ & $10 \%$ & $10 \%$ & $4 \%$ & $4 \%$ \\
\hline $80 \mathrm{mg}$ & $0 \%$ & $0 \%$ & $7 \%$ & $6 \%$ \\
\hline \multicolumn{5}{|l|}{ Rosuvastatin } \\
\hline $10 \mathrm{mg}$ & $15 \%$ & $16 \%$ & & \\
\hline $20 \mathrm{mg}$ & $13 \%$ & $12 \%$ & & \\
\hline $40 \mathrm{mg}$ & $0 \%$ & $0 \%$ & & \\
\hline
\end{tabular}

Note: Proportions are relative to the number of patients prescribed each initial dose. 
Table 5 Mean lipid panel changes at years one and five relative to baseline measurement

\begin{tabular}{lllll}
\hline $\begin{array}{l}\text { Time point/initial } \\
\text { treatment }\end{array}$ & LDL-C & TC & HDL-C & TC/HDL-C \\
\hline Year I & & & & \\
$\quad$ Simvastatin & $-30 \%$ & $-22 \%$ & $5 \%$ & $-25 \%$ \\
$\quad$ Atorvastatin & $-34 \%$ & $-26 \%$ & $4 \%$ & $-28 \%$ \\
$\quad$ Rosuvastatin & $-38 \%$ & $-27 \%$ & $7 \%$ & $-31 \%$ \\
Year 5 & & & & \\
$\quad$ Simvastatin & $-34 \%$ & $-25 \%$ & $5 \%$ & $-28 \%$ \\
$\quad$ Atorvastatin & $-39 \%$ & $-29 \%$ & $4 \%$ & $-32 \%$ \\
$\quad$ Rosuvastatin & $-44 \%$ & $-31 \%$ & $7 \%$ & $-36 \%$ \\
\hline
\end{tabular}

Abbreviations: HDL-C, high-density lipoprotein cholesterol; LDL-C, low-density lipoprotein cholesterol; TC, total cholesterol.

\section{Endpoints}

At 5 years, the Kaplan-Meier estimated MACE incidence was $4.9 \%$ for initial treatment with simvastatin, $4.4 \%$ for atorvastatin, and $4.1 \%$ for rosuvastatin. At 10 years, the corresponding incidence of MACE was $10.0 \%, 9.2 \%$, and $8.4 \%$, respectively. The absolute difference in MACE incidence generally increased with time (Figure 2).

At 5 years, the RR of MACE was 0.906 (95\% confidence interval: $0.888-0.923 ; P<0.001)$ for initial treatment with atorvastatin rather than simvastatin, 0.831 (0.812-0.850; $P<0.001)$ for rosuvastatin rather than simvastatin, and $0.918(0.898-0.938 ; P<0.001)$ for rosuvastatin rather than atorvastatin. At 10 years, the corresponding RRs of MACE were $0.917(0.906-0.929 ; P<0.001)$ for atorvastatin rather than simvastatin, $0.843(0.830-0.856 ; P<0.001)$ for rosuvastatin rather than simvastatin, and $0.919(0.906-0.932$; $P<0.001)$ for rosuvastatin rather than atorvastatin (RR values less than one are favorable to the treatment listed

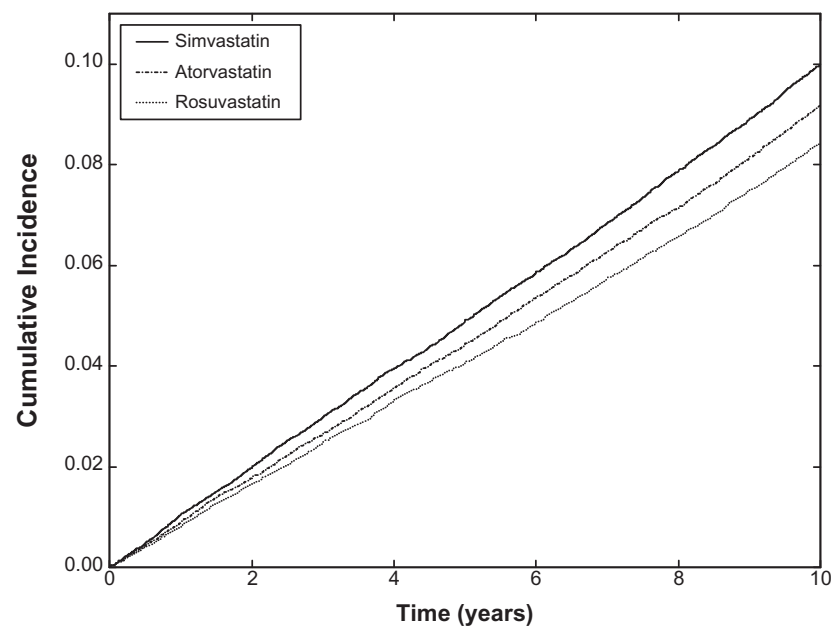

Figure 2 The Kaplan-Meier cumulative incidence of major adverse cardiovascular events in the full population. first in each comparison above). RR was roughly equivalent across the embedded subgroups, with a slight decrease in benefit (RR closer to one) for the higher-risk groups. An overview of RR is shown in Figures 3-5.

At 5 years, the NNT to prevent one MACE was 217 for initial treatment with atorvastatin rather than simvastatin, 122 for rosuvastatin rather than simvastatin, and 275 for rosuvastatin rather than atorvastatin. At 10 years, the NNT to prevent one MACE was 122 for initial treatment with atorvastatin rather than simvastatin, 64 for rosuvastatin rather than simvastatin, and 135 for rosuvastatin rather than atorvastatin. The NNT figures trended lower among higherrisk subgroups and were lowest in the subgroup with prior CVD, with 5-year figures of 80 for treatment with atorvastatin rather than simvastatin, 45 for rosuvastatin rather than simvastatin, and 105 for rosuvastatin rather than atorvastatin. An overview of NNT is shown in Figure 6.

\section{Sensitivity analysis}

The high-titration case showed a small decrease in the incidence of MACE compared with that in the main study, and the low-titration case showed a slightly increased incidence of MACE compared with that in the main study. Shown together in Table 6, the point estimates of RR of MACE at year five, comparing the low- and high-intensification

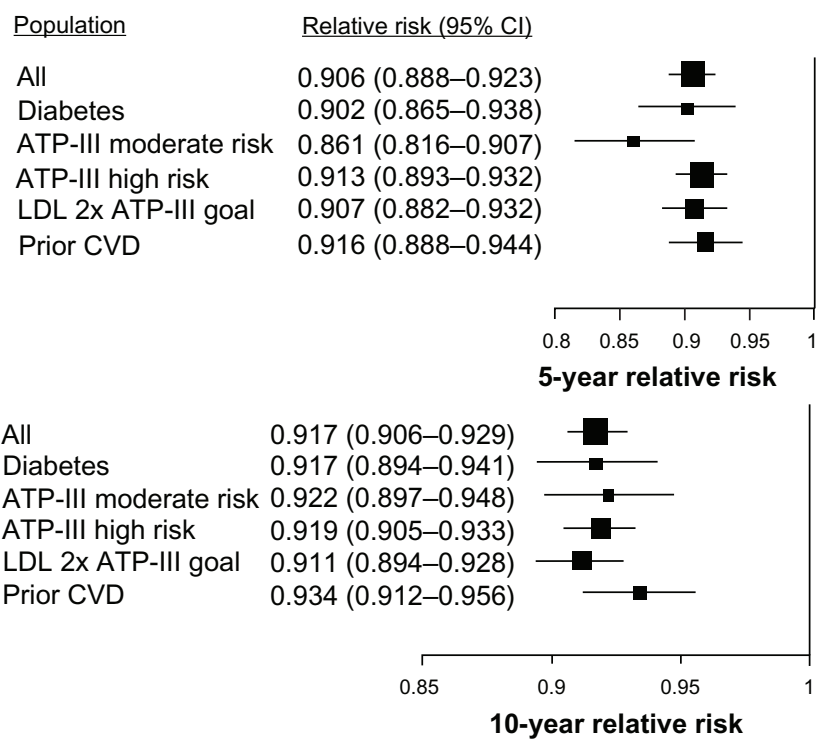

Figure 3 The relative risk of major adverse cardiovascular events for initial treatment with atorvastatin rather than simvastatin for each subpopulation at 5 years and 10 years.

Notes: The size of each black square is approximately proportionate to the number of patients who had a major adverse cardiovascular event; horizontal lines indicate $95 \%$ confidence intervals; a number $<\mathrm{I}$ is favorable to initial treatment with atorvastatin. Abbreviations: ATP-III, Adult Treatment Panel III; Cl, confidence interval; CVD, cardiovascular disease; LDL, low-density lipoprotein. 

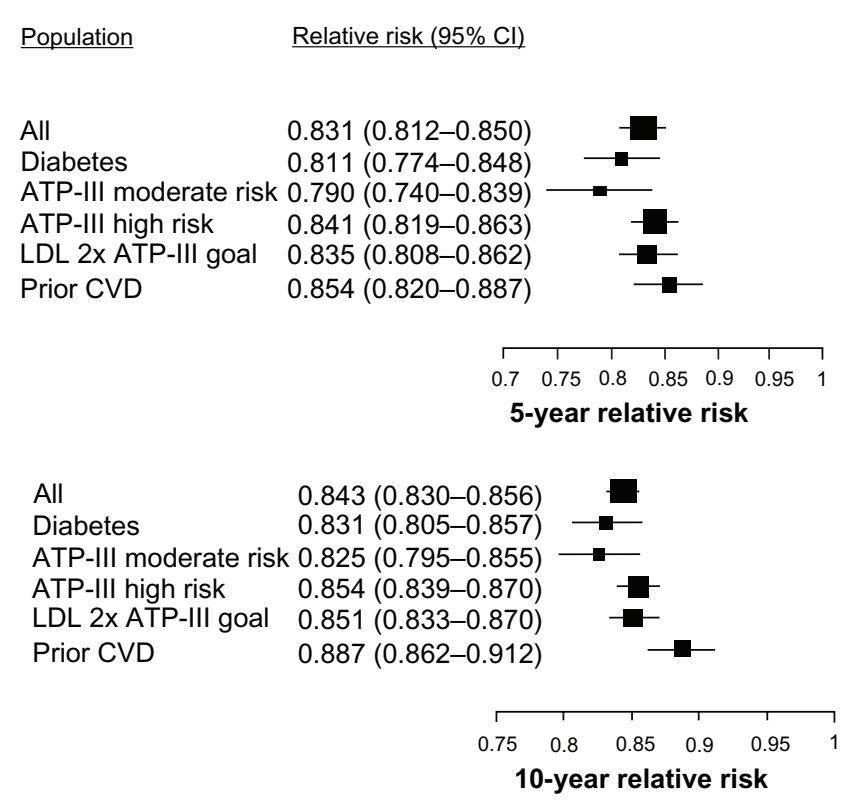

Figure 4 The relative risk of major adverse cardiovascular events for initial treatment with rosuvastatin rather than simvastatin for each subpopulation at 5 years and 10 years.

Notes: The size of each black square is approximately proportionate to the number of patients who had a major adverse cardiovascular event; horizontal lines indicate $95 \%$ confidence intervals; a number $<\mathrm{I}$ is favorable to initial treatment with rosuvastatin. Abbreviations: ATP-III, Adult Treatment Panel III; Cl, confidence interval; CVD, cardiovascular disease; LDL, low-density lipoprotein.

cases crossed with the three treatment scenarios, illustrate the sensitivity of the RR comparisons to treatment intensification rates. In three cases, RRs greater than one were observed, indicating at least some sensitivity to the titration rates used for this study. However, these cases are rather extreme in that they compare exposure groups having very low titration rates with control groups having very high titration rates. In cases in which intensification rates were varied across all three treatment scenarios uniformly, little change in RR between treatment scenarios was observed.

In the atorvastatin-only scenario, the 5- and 10-year RRs of MACE for treatment with rosuvastatin rather than atorvastatin were slightly lower than in the main simulation: 0.900 (95\% confidence interval: $0.881-0.919 ; P<0.001$ ) and $0.911(0.899-0.924 ; P<0.001)$, respectively. The corresponding 5 - and 10-year NNTs were also slightly lower: 218 and 120 , respectively.

\section{Discussion}

This simulated study compared the relative effectiveness of three different statin titration protocols, each containing the most potent statin doses currently available, across a range of populations with varying cardiovascular risk.
Population

All

Diabetes

ATP-III moderate risk

ATP-III high risk

LDL 2x ATP-III goal

Prior CVD

All

Diabetes

ATP-III moderate risk

ATP-III high risk

LDL 2x ATP-III goal

Prior CVD
Relative risk $(95 \% \mathrm{Cl})$

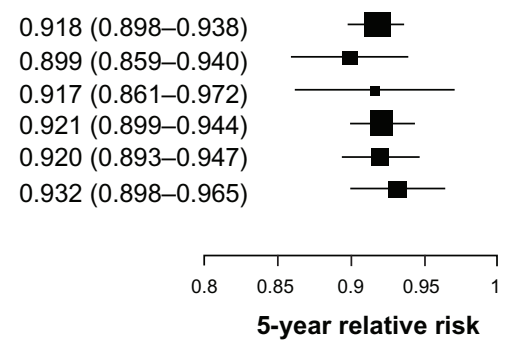

$0.919(0.906-0.932)$ $0.906(0.880-0.931)$

$0.894(0.865-0.923)$

$0.930(0.915-0.945)$

$0.935(0.916-0.954)$

$0.950(0.926-0.973)$

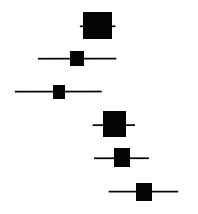

$0.85-0.9-0.95-1$

10-year relative risk

Figure 5 The relative risk of major adverse cardiovascular events for initial treatment with rosuvastatin rather than atorvastatin for each subpopulation at 5 years and 10 years.

Notes: The size of each black square is approximately proportionate to the number of patients who had a major adverse cardiovascular event; horizontal lines indicate $95 \%$ confidence intervals; a number less than I is favorable to initial treatment with rosuvastatin.

Abbreviations: ATP-III, Adult Treatment Panel III; Cl, confidence interval; CVD, cardiovascular disease; LDL, low-density lipoprotein.

Despite the availability of powerful statins, a large body of clinical evidence demonstrating the safety and efficacy of statins, and national guidelines recommending statins, many patients remain undertreated and fail to reach their lipid goals. ${ }^{4,5}$ The strategy of initiating patients on higher starting doses (chosen according to individual patient lipid levels, goal, and cardiovascular risk profile) appears a promising alternative to the more widely practiced "start low and titrate" approach. ${ }^{21}$

The relative benefit of treatment between the most aggressive and most conservative scenario - rosuvastatin and simvastatin - varied modestly across all populations considered. For example, the 5-year RR of MACE given initial treatment with rosuvastatin versus simvastatin varied only from 0.790 (95\% confidence interval: 0.740 0.839) for those with moderate ATP-III risk (who had the lowest incidence of MACE) to 0.854 (0.820-0.887) for those with prior CVD (who had the highest incidence of MACE). The potential absolute benefit was most compelling among patients in the high-risk subpopulations: those with previously diagnosed CVD, those deemed "high risk" under the ATP-III guidelines, and those with LDL-C more than double their aggressive ATP-III goal. In the rosuvastatin versus simvastatin comparison, 


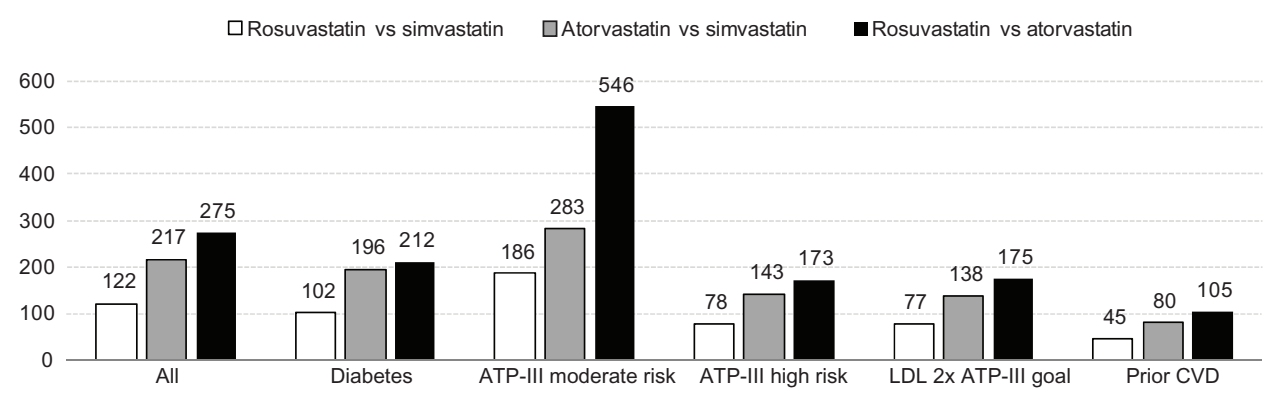

Figure 6 The number needed to treat to prevent one major adverse cardiovascular event at 5 years, according to baseline population.

Note: Smaller positive values indicate greater absolute benefit due to the treatment listed first in the legend.

Abbreviations: ATP-III, Adult Treatment Panel III; CVD, cardiovascular disease; LDL, low-density lipoprotein; vs, versus.

the 5-year NNTs for these groups were 45, 78, and 77, respectively. At the 10-year time point, the corresponding NNTs were 35, 44, and 43, respectively. Among those with prior CVD, the 5-year NNT was 138 for treatment with atorvastatin rather than simvastatin and 175 for treatment with rosuvastatin rather than atorvastatin. These results indicate a significant opportunity to reduce the mediumand long-term incidence of MACE among patients at high cardiovascular risk by drawing from more aggressive formularies when initiating statin therapy. Compared with the main simulation, the atorvastatin-only simulation resulted in a minor change in $\mathrm{RR}$, which seems to indicate that given the observed rates of treatment intensification in the real world, the availability of the most effective statin dose in follow-up care appears to play a minor role in cardiovascular risk reduction when compared with the efficacy of the initial dose.

\section{Limitations}

This study is based on a mathematical model and is subject to all the assumptions used to create it. The treatments examined in this study were modeled using results from published trials, and substantial efforts were taken to ensure the applicability of this simulation to actual clinical care settings by calibrating intervention effects and the patterns of statin use to real-world titration patterns. However, some aspects of real-world lipid management are not captured in the simulation. The analysis of pharmaceutical claims data used for calibration revealed a small but significant fraction of people backing off from their initially prescribed therapy, and the simplified titration protocols used for these simulations do not account for adverse side effects or backward titration, which potentially affects the degree to which this study reflects real-world clinical care scenarios. Although analysis revealed a minor sensitivity to large changes in individual intensification probabilities, even the extreme cases of the sensitivity analysis demonstrated a treatment benefit associated with aggressively initiating statin therapy. Furthermore, changes in intensification rates that affected all three treatment scenarios uniformly resulted in small changes in the relative benefit associated with the more aggressive treatment scenarios. Hence, it appears unlikely that reasonable differences between the intensification patterns used for these simulations and those that exist in the real world would significantly affect the findings of this study.

Table 6 Comparison of initial treatment and sensitivity analysis scenarios using relative risk of major adverse cardiovascular events at year five to measure benefit

\begin{tabular}{|c|c|c|c|c|c|c|}
\hline \multirow{2}{*}{$\begin{array}{l}\text { Initial treatment/intensification } \\
\text { likelihood }\end{array}$} & \multicolumn{3}{|c|}{ Atorvastatin (exposure) } & \multicolumn{3}{|c|}{ Rosuvastatin (exposure) } \\
\hline & Low & Base & High & Low & Base & High \\
\hline \multicolumn{7}{|l|}{ Simvastatin (control) } \\
\hline Low & 0.924 & 0.867 & 0.788 & 0.864 & 0.796 & 0.723 \\
\hline Base & 0.964 & 0.906 & 0.822 & 0.902 & 0.831 & 0.755 \\
\hline High & 1.051 & 0.987 & 0.897 & 0.983 & 0.906 & 0.823 \\
\hline \multicolumn{7}{|l|}{ Atorvastatin (control) } \\
\hline Low & & & & 0.936 & 0.862 & 0.783 \\
\hline Base & & & & 0.996 & 0.918 & 0.834 \\
\hline High & & & & 1.097 & 1.011 & 0.918 \\
\hline
\end{tabular}

Note: Relative risk $<$ I favors the control group, whereas relative risk $>$ I favors the exposure group. 


\section{Conclusion}

This simulated study found that the practice of initializing statin therapy aggressively rather than conservatively has strong potential to improve cardiovascular risk for patients with elevated cholesterol. Among patients beginning statin therapy, the choice of the initial treatment appears to have a significant impact on patients' medium- and long-term cardiovascular risk. Under a treatment strategy of conservative initial assignment, patients did not appear to intensify treatment in sufficiently large numbers or quickly enough to lower cardiovascular risk as much as occurred when using a stronger set of initial treatments, despite the fact that the strongest statin doses were available in every treatment scenario. Furthermore, the availability of the most effective statin doses appears to play a minor role in reducing cardiovascular risk, when compared with the benefit of initiating therapy with stronger statins. This indicates a compelling real-world opportunity to reduce the incidence of cardiovascular events by initiating therapy using more aggressive treatments. In absolute terms, patients at highest cardiovascular risk would likely benefit the most from such a strategy.

\section{Acknowledgment}

This research was supported by funding from AstraZeneca Pharmaceuticals LP.

\section{Disclosure}

Andrew van Herick, C Andy Schuetz, and Peter Alperin are employees of Archimedes, Inc, and were paid consultants to AstraZeneca in connection with the development of the manuscript. Michael Bullano, Sanjeev Balu, and Sanjay Gandhi are employees of AstraZeneca.

\section{References}

1. Mackay J, Mensah GA. The Atlas Of Heart Disease And Stroke. Geneva: World Health Organization; 2004. Available from: http://www.who.int/ cardiovascular_diseases/resources/atlas/en/. Accessed January 20, 2012.

2. Grundy SM, Cleeman JI, Merz CN, et al. Implications of recent clinical trials for the National Cholesterol Education Program Adult Treatment Panel III guidelines. Circulation. 2004;110(2):227-239.

3. Baigent C, Keech A, Kearney PM, et al. Efficacy and safety of cholesterol-lowering treatment: prospective meta-analysis of data from 90,056 participants in 14 randomised trials of statins. Lancet. 2005;366(9493):1267-1278.

4. Willey VJ, Bullano MF, Shoetan NN, Gandhi SK. Therapy modifications and low-density lipoprotein cholesterol goal attainment rates associated with the initiation of generic simvastatin. Curr Med Res Opin. 2010;26(1):121-128.

5. Waters DD, Brotons C, Chiang CW, et al. Lipid treatment assessment project 2: a multinational survey to evaluate the proportion of patients achieving low-density lipoprotein cholesterol goals. Circulation. 2009;120(1):28-34.
6. Eddy DM, Schlessinger L. Validation of the archimedes diabetes model. Diabetes Care. 2003;26(11):3102-3110.

7. Eddy DM, Schlessinger L. Archimedes: a trial-validated model of diabetes. Diabetes Care. 2003;26(11):3093-3101.

8. National Health and Nutrition Examination Survey data 1999-2006 [webpage on the Internet]. Atlanta, GA: Centers for Disease Control and Prevention [updated June 19, 2012]. Available from: http://www. cdc.gov/nchs/nhanes.htm. Accessed January 20, 2012.

9. Heart Protection Study Collaborative Group. MRC/BHF Heart Protection Study of cholesterol lowering with simvastatin in 20,536 high-risk individuals: a randomised placebo-controlled trial. Lancet. 2002;360(9326):7-22.

10. Randomised trial of cholesterol lowering in 4444 patients with coronary heart disease: the Scandinavian Simvastatin Survival Study (4S). Lancet. 1994;344(8934):1383-1389.

11. Pedersen TR, Faergeman O, Kastelein JJ, et al. High-dose atorvastatin vs usual-dose simvastatin for secondary prevention after myocardial infarction: the IDEAL study: a randomized controlled trial. JAMA. 2005;294(19):2437-2445.

12. Colhoun HM, Betteridge DJ, Durrington PN, et al. Primary prevention of cardiovascular disease with atorvastatin in type 2 diabetes in the Collaborative Atorvastatin Diabetes Study (CARDS): multicentre randomised placebo-controlled trial. Lancet. 2004;364(9435): 685-696.

13. LaRosa JC, Grundy SM, Waters DD, et al. Intensive lipid lowering with atorvastatin in patients with stable coronary disease. $N$ Engl J Med. 2005;352(14):1425-1435.

14. Schlessinger L, Eddy DM. Archimedes: a new model for simulating health care systems - the mathematical formulation. J Biomed Inform. 2002;35(1):37-50.

15. Expert Panel on Detection, Evaluation, and Treatment of High Blood Cholesterol in Adults. Executive summary of the Third Report of The National Cholesterol Education Program (NCEP) Expert Panel on Detection, Evaluation, And Treatment of High Blood Cholesterol In Adults (Adult Treatment Panel III). JAMA. 2001;285(19): 2486-2497.

16. United States Department of Health and Human Services. JNC 7 Express: The Seventh Report of the Joint National Committee on Prevention, Detection, Evaluation, and Treatment of High Blood Pressure. Bethesda, MD: National Institutes of Health; 2003.

17. Nathan DM, Buse JB, Davidson MB, et al. Management of hyperglycemia in type 2 diabetes: a consensus algorithm for the initiation and adjustment of therapy update regarding thiazolidinediones: a consensus statement from the American Diabetes Association and the European Association for the Study of Diabetes. Diabetes Care. 2008;31(1): 173-175.

18. Schuetz CA, van Herick A, Alperin P, Peskin B, Hsia J, Gandhi S. Comparing the effectiveness of rosuvastatin and atorvastatin in preventing cardiovascular outcomes: estimates using the Archimedes Model. J Med Econ. June 20, 2012. [Epub ahead of print.]

19. Jones PH, Davidson MH, Stein EA, et al. Comparison of the efficacy and safety of rosuvastatin versus atorvastatin, simvastatin, and pravastatin across doses (STELLAR* Trial). Am J Cardiol. 2003;92(2): $152-160$.

20. Altman DG, Andersen PK. Calculating the number needed to treat for trials where the outcome is time to an event. BMJ. 1999;319(7223): 1492-1495.

21. Jones PH. Statins: the case for higher, individualized starting doses. Cleve Clin J Med. 2005;72(9):811-816

22. Cannon CP, Braunwald $\mathrm{E}, \mathrm{McCabe} \mathrm{CH}$, et al. Intensive versus moderate lipid lowering with statins after acute coronary syndromes. $N$ Engl J Med. 2004;350(15):1495-1504.

23. Ridker PM, Danielson E, Fonseca FA, et al. Rosuvastatin to prevent vascular events in men and women with elevated $\mathrm{C}$-reactive protein. $N$ Engl J Med. 2008;359(21):2195-2207. 
24. Sever PS, Dahlof B, Poulter NR, et al. Prevention of coronary and stroke events with atorvastatin in hypertensive patients who have average or lower-than-average cholesterol concentrations, in the Anglo-Scandinavian Cardiac Outcomes Trial - Lipid Lowering Arm (ASCOT-LLA): a multicentre randomised controlled trial. Lancet. 2003;361(9364):1149-1158.
25. Sacks FM, Pfeffer MA, Moye LA, et al. The effect of pravastatin on coronary events after myocardial infarction in patients with average cholesterol levels. Cholesterol and recurrent Events Trial investigators. N Engl J Med. 1996;335:1001-1009.

\section{Publish your work in this journal}

ClinicoEconomics \& Outcomes Research is an international, peerreviewed open-access journal focusing on Health Technology Assessment, Pharmacoeconomics and Outcomes Research in the areas of diagnosis, medical devices, and clinical, surgical and pharmacological intervention. The economic impact of health policy and health systems organization also constitute important areas of coverage. The manuscript management system is completely online and includes a very quick and fair peer-review system, which is all easy to use. Visit http://www.dovepress.com/testimonials.php to read real quotes from published authors.

Submit your manuscript here: http://www.dovepress.com/clinicoeconomics-and-outcomes-research-journal 